\title{
Designing Gesture-Based Control for Factory Automation
}

\author{
Tomi Heimonen ${ }^{1}$, Jaakko Hakulinen ${ }^{1}$, Markku Turunen ${ }^{1}$, Jussi P.P. Jokinen ${ }^{2}$, \\ Tuuli Keskinen ${ }^{1}$, and Roope Raisamo ${ }^{1}$ \\ ${ }^{1}$ School of Information Sciences, University of Tampere, Finland \\ \{tomi.heimonen, jaakko.hakulinen, markku.turunen, \\ tuuli.keskinen, roope.raisamo\} @sis.uta.fi \\ ${ }^{2}$ Department of Computer Science and Information Systems, University of Jyväskylä, Finland \\ jussi.p.p.jokinen@jyu.fi
}

\begin{abstract}
We report the development and evaluation of a gesture-based interaction prototype for controlling the loading station of a factory automation system. In this context, gesture-based interaction has the potential to free users from the tedious physical controls but it must also account for safety considerations and users' perceptions. We evaluated the gesture interaction concept in the field to understand its applicability to industrial settings. Our findings suggest that gesture-based interaction is an emotional, physically charged experience that has the potential to enhance the work process. Participants' feedback also highlighted challenges related to the reliability of gesture recognition technology in the workplace, the perceived professionalism of gesture-based interaction, and the role of physical feedback in promoting feeling of control. Our results inform the development of gesture-based interaction for similar contexts.
\end{abstract}

Keywords: Gesture-based interaction, emotions, user experience, field study.

\section{$1 \quad$ Introduction}

Gesture-based interaction could provide many benefits as an alternative to physical controls in industrial settings, where the manual operation of machinery can be ergonomically challenging and hazardous or is governed by safety regulations. Several characteristics of gesture-based systems affect their usability in such settings, including technical aspects such as latency of operation, stability, resolution, and precision [1], as well as human-centered aspects such as intuitiveness, ergonomics, and ease of gesture performance and recall [2]. Appropriately designed systems must balance these sometimes contradictory concerns.

We report the findings from a year-long design and development process of a gesture-based interaction concept, focusing on the results of evaluations conducted in real context of use. The overall goal of the research was to create novel interaction concepts for metal workshops and to study their potential in real work tasks. Our research was aimed at answering the following questions: 
- Which factors affect the acceptability of gesture control in industrial settings?

- What is the user experience of gesture control in industrial settings, especially with respect to its emotional aspects?

\section{Related Work}

\subsection{Gesture-Based Interaction}

Gesture tracking technologies can be broadly divided into perceptual (utilizing, e.g., vision and audio) and non-perceptual (based on, e.g., mouse or touch surfaces) [3]. The taxonomy proposed by Karam and schraefel [3] divides gesture styles between deictic (pointing), manipulative (tight coupling between the controlled object and hand/arm movement), semaphoric (symbolic), gesticulation (combination of gesture and speech), and language gestures (e.g., sign languages). Due to the prevalence of symbolic gesturing in the real world (e.g., police and military hand signals), we chose it as the starting point for our design.

Empty-handed, or touchless, gestural interaction has been proposed to be advantageous in various real world contexts, such as medical environments and environments where physical input devices could be vandalized [4]. In our context of use, the situation is similar to the latter, as introducing input devices into the factory environment is not viable. For example, touchscreens could be smeared with residues, and remote controls could easily be damaged or misplaced. Examples of perceptual gesture-based interaction in a context of use resembling ours can be found in human-robot interaction [5-7]. However, to our knowledge, our work is one of the first user-centered studies of designing gesture-based control to operate factory automation systems.

\subsection{Designing Gesture Interactions}

Combining the technical and user-centered requirements makes the design of gesture interaction challenging. Although it may be possible to design an efficient and easy to recognize gesture vocabulary for human-machine interaction, one needs to also consider the social implications of gesturing in the workplace. Previous research suggests that gestures that are based on subtle movements and are similar to existing technology and every day actions are preferred to gestures that look uncommon, are physically uncomfortable, or interfere with face-to-face conversations [8]. Additionally, the general meaning of the gesture should be understandable to both the user and bystanders to be socially acceptable [9]. Early stage development that looks past technical restrictions is one way to avoid expending effort on gestures that are not acceptable [8]. However, as Wobbrock et al. [10] point out, reliability of recognition is nevertheless an important criterion for early prototypes. Our design approach is a hybrid of the technology-oriented and human-centered styles; we aimed for reliable recognition through designed gestures, but carried out a series of design workshops to ensure the gestures are also acceptable. 


\section{Gesture-Based Interaction for Factory Automation}

The context of use for the proposed gesture interaction concept is the loading station area, which is a part of a manufacturing automation system (Figure 1). The operator's task is to attach raw materials to a pallet using various tools, which the automation system then moves to machining, and subsequently to remove the machined parts. Using a button panel situated to the side of the loading station, the operator can open and close the door separating the automated storage from the operating area, drive the pallet in or out of the storage, and rotate the pallet in either horizontal direction. The emergency stop can also be used at any time.

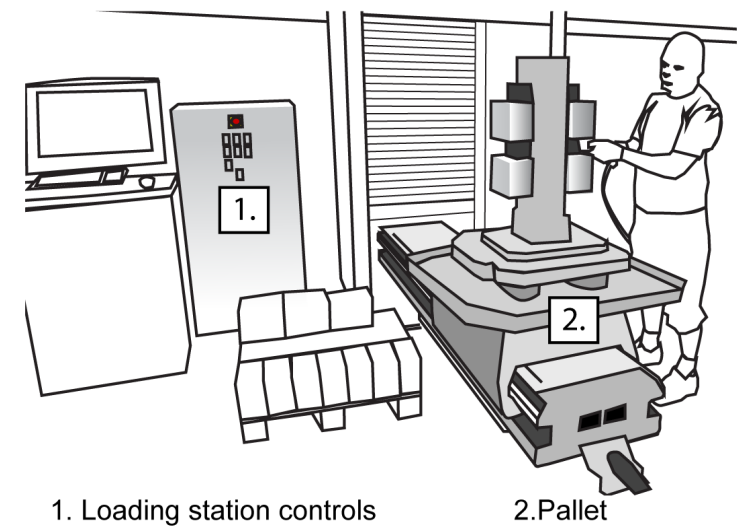

Fig. 1. User attaching materials to the pallet (original image courtesy of Fastems)

The loading station controls are placed away from the pallet due to safety regulations. In crowded workshop conditions, the controls can be hard to reach, and their operation requires constant movement from the pallet to the controls and back. These issues could be alleviated with gestures, as the loading station could be controlled by simply taking a step back to a safe distance from the machine and performing the appropriate gesture, even while grasping tools. The use of gestures would naturally also require strong safeguards, such as multiple means of tracking the operator's location with respect to the machine.

\subsection{Gesture Vocabulary for Loading Station Control}

The gesture vocabulary was designed in a series of workshops with representatives from factory automation manufacturing industry to ensure its validity in the realistic industrial context. This collaboration was grounded on prior user observations in industrial environments. During the design process we iterated different versions of the gesture vocabulary and visual feedback to ensure the operation was robust and the feedback informative. This process resulted in a gesture set of seven poses (Figure 2). The gestures were designed to be distinctive from one another, so that their recognition would be as reliable as possible using the template-based recognition 
algorithm implemented for the prototype. The poses also attempt to provide intuitive mappings to the functioning of the loading station through body-centric associations. For example, driving the pallet in and out is mapped to the user's horizontally extended arm, while door operation is mapped to vertically extended arm, both matching the direction of the resulting movement.

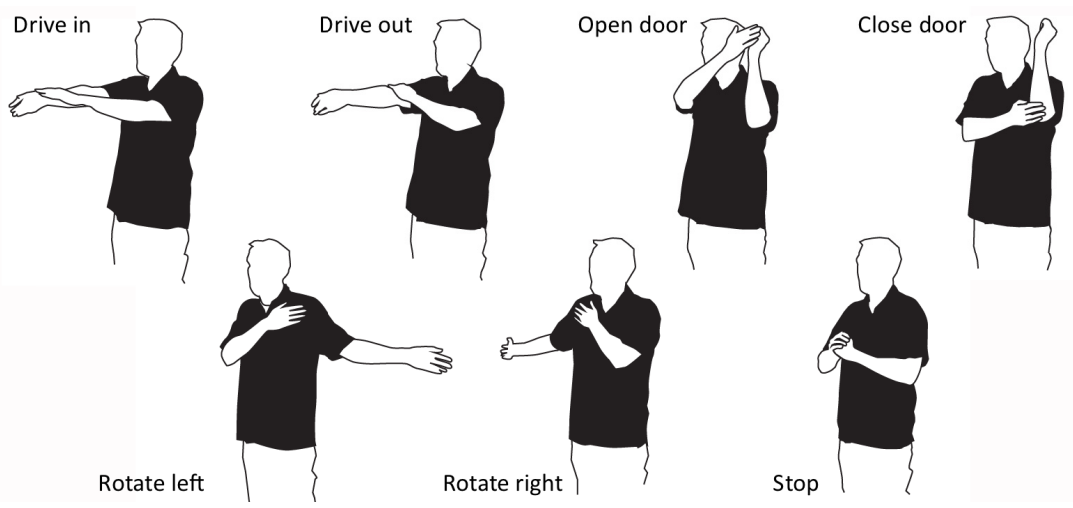

Fig. 2. The proposed gesture set consisting of static hand and arm poses

\subsection{Loading Station Simulator}

A simulator application was implemented to demonstrate the concept. It contains a 3D model of the loading station and an instruction screen, which would be visible on a separate display in a real world set up. Figure 3 shows the screens as displayed during the field tests. The instruction screen has icons, which depict the pose for each operation and indicate their activation. It provides continuous feedback on system state and gesture recognition, and proved valuable in letting users know that the system is successfully tracking their movements. We utilized the Microsoft Kinect sensor via the OpenNI framework (http://www.openni.org) in early versions of the prototype, and the official Kinect SDK in later iterations.

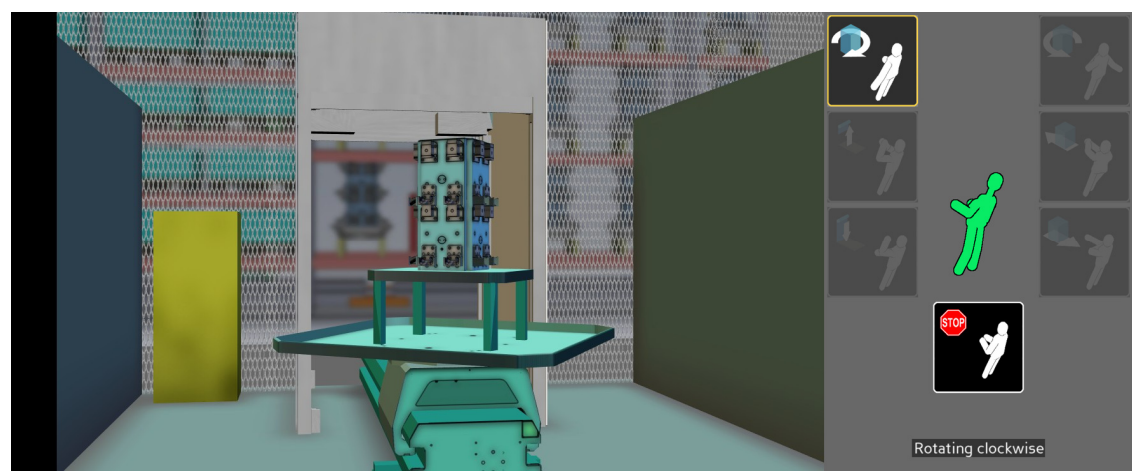

Fig. 3. Loading station simulator (left) and the gesture instruction screen (right) 


\section{$4 \quad$ Evaluating Gesture-Based Interaction in Real Contexts of Use}

The emotional user experience and acceptance of the concept were evaluated in real workshop environment. The first study was conducted at a factory automation manufacturing site and the second at a local metal workshop, where loading stations similar to the simulated one are used daily. Externally, the prototype was similar in both studies, but internal data processing was updated to improve stability.

\subsection{Method}

Participants were opportunistically recruited on-site. A total of 22 participants, 2 female and 20 male, took part in the evaluation. They had an average work experience with factory automation loading stations of 6.0 years (range $=0-25$ years, s.d. $=6.9$ ). Participant backgrounds ranged from factory automation product designers to assembly line workers and metal workshop laborers.

After a demonstration of the gesture commands by a researcher, the participants were able to briefly practice the gestures. Next, they were asked to perform each of the gestures twice according to a script cued by a researcher, simulating the operation of a real loading station. Interaction took place in front of the simulator, which was presented on a 37-inch high definition television next to the loading station cell.

User experience was measured with a questionnaire, which contained a Likert scale of eleven questions concerning emotional experiences including alertness, anxiety, delight, desperation, determination, excellence, frustration, pride, skillfulness, success, and surprise. In addition, an item concerning the feeling of control was included. Another Likert scale of five statements concerned the acceptability of the gestures: their practicality, necessity, professionalism, intelligence, and physical demand. The questionnaire was filled after the participants had tested the prototype. In addition, short interviews were conducted with nine of the participants.

\subsection{Results}

Emotional User Experience. The responses to the questionnaire were analyzed using multidimensional scaling (MDS). The resulting configuration shows which emotions are related to each other (Figure 4). The assumption is that similar emotional items are correlated with the same underlying user experience factor, which can be calculated as their sum variable. The items were separated by their emotional valence into pleasant $(\alpha=.862)$ and unpleasant $(\alpha=.804)$ emotional groups, along the $x$-axis. Cronbach alphas over 0.8 were taken as a confirmation that the items were reliable indicators of the underlying emotional factor.

The results indicate the emergence of two distinct emotional user experience factors: competence [11] and frustration [12]. Competence, or self-efficacy, is linked to the participants being pleasantly surprised by the new suggested interface (Spearman $\rho=.771, \mathrm{p}<.001$ ), and associating feeling of control with very positive emotional experiences, such as pride and success. In addition, a positive association between having a pleasant user experience and feeling in control was observed $(\rho=.508$, 
$\mathrm{p}=.019$ ). Frustration was evident in even slightly negative, or unpleasant, user experience being strongly associated with not accepting the gestures. Accordingly, negative correlations were observed between feeling frustration and perceiving the gestures as intelligent $(\rho=-0.542, p=0.011)$, professional $(\rho=-0.587, p=0.005)$, safe $(\rho=-0.671, p=0.001)$, and needful $(\rho=-0.509, p=0.018)$.

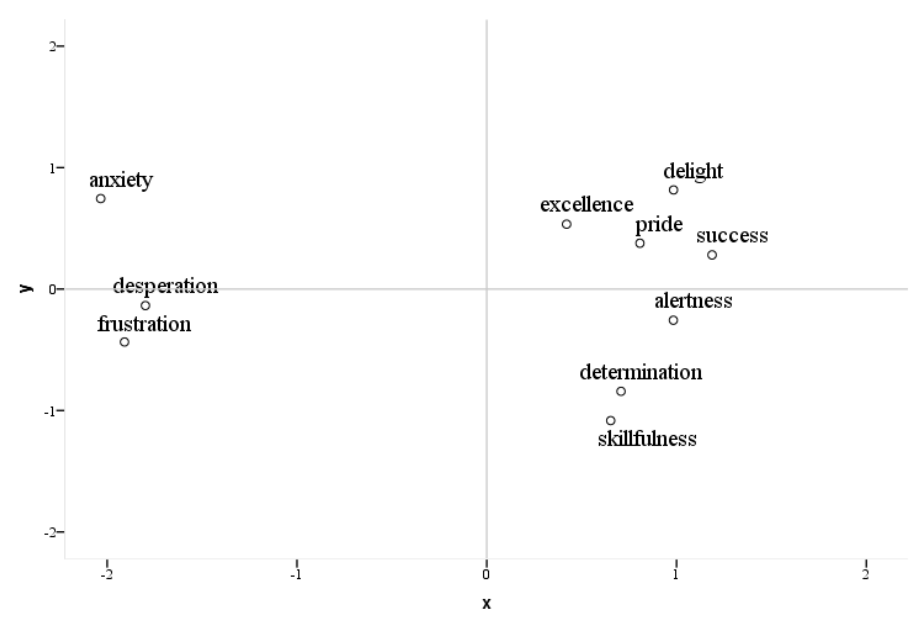

Fig. 4. Multidimensional scaling solution of experienced emotions. The configuration shows that two groups are formed along the x-axis, which indicates the valence of the emotions.

Interview Feedback. The interviews were conducted with metal workshop workers (4 respondents) that operate loading stations daily, and factory automation mechanics (5) that assemble the loading stations. Most felt that the new scheme of interaction was a welcome introduction to the usual work routine. The notable benefits mentioned were the increased freedom of movement, speeding up the work process, improving safety, and easing the learning process of the control interface due to easily learnable gestures. However, many also suggested that the current interface is more reliable, and the shift to non-physical controls would introduce errors without making them easier. Four notable items of doubt were extracted from the interviews:

1. Can the operator trust the delicate sensory in a factory environment?

2. How easily does the sensor break or get dirty?

3. Is it safe to perform elaborate gestures in factory environment?

4. The control interface is already easy. Would it truly be easier to perform gestures instead of walking to the control station to press a button?

When asked about the perceived professionalism of the proposed user interface, the participants made references to attributes not welcome in industrial environment such as "games" and "fooling around". Mostly these references were associated with the gestures themselves: moving arms and body around was perceived as being something not suitable in a factory environment, which suggests gesture control as currently designed might break social norms at the workplace. Physical controls also seem to significantly affect the feeling of professionalism. The most important factor 
associated with feeling of control was the lack of physical contact with the interface. Comments such as "it felt strange that I couldn't touch it", "I'm used to getting direct physical feedback from the button", and "I feel more in control with the traditional physical buttons" indicated this to be one of the critical factors influencing the acceptance of the new gesture-based interface.

\section{Discussion}

Our research contributes to the development of gesture-based interaction for industrial workplaces. First, we found that the proposed gesture interface evoked clear emotional experiences as distinct groups of pleasant and unpleasant emotions were observed. These were shown to be associated with feeling of control and acceptance of the concept. In designing gesture-based interaction, it is thus critical to actively support the feelings of self-efficacy and minimize the experienced frustration.

Second, we identified doubts related to the professionalism of gesture-based interaction that are strongly linked to the absence of physical control and the social acceptability of gesturing in the workplace. The first aspect is closely associated with feeling self-efficant: is it possible to feel competent and in control without physical interaction? This resonates with findings from previous research, which suggest that it is important to understand the relationship between the physicality of control and the system being used, and how this affects the users' preferences [13]. In this respect, an obvious shortcoming of touchless interaction is the lack of tactile feedback, and one must provide a form of feedback that promotes the feeling of control when physical controls are absent. With respect to acceptability, it should be noted that the focus of the research was to develop and study novel interaction concepts for future work settings. It is an open question to what extent the concerns with professionalism apply to new generations of users who already have experiences with using gestures in games and with mobile devices.

Finally, results from studies that are not a part of the users' work practice need to be interpreted with care. Benefits and drawbacks with respect to existing interfaces are difficult to estimate without experiencing gesture-based interaction in the context of real work tasks. For example, would the audiovisual feedback from a real loading station compensate for the lack of physical control? Longitudinal, in situ studies of gesture-based production systems are needed to address the above issues.

\section{Conclusion}

Gesture-based interaction in industrial setting is a novel and physically charged activity that is also an emotional experience. When designing gesture-based interaction for industrial environments, attention should be paid to creating a system that promotes self-efficant experiences. An optimal interaction is challenging but practical in order to make the interaction an emotionally pleasurable and novel experience. Challenges to address in future work are related to studying the perceived robustness of the gesture tracking technology in real work tasks, designing the physical gesture movements to fit the work context, and compensating for the lack of physical control with alternative feedback methods. 
Acknowledgements. This research was carried out as part of the Finnish Metals and Engineering Competence Cluster (FIMECC)'s UXUS program. Appreciation is extended to Matti Nurminen and colleagues from Fastems Oy Ab, Tuomas Kiviluoto from the Agora Center at the University of Jyväskylä, and Mika Käki from Idean.

\section{References}

1. Crowley, J.L., Coutaz, J., Bérard, F.: Perceptual User Interfaces: Things That See. Commun. ACM 43(3), 54-64 (2000)

2. Nielsen, M., Störring, M., Moeslund, T.B., Granum, E.: A Procedure for Developing Intuitive and Ergonomic Gesture Interfaces for HCI. In: Camurri, A., Volpe, G. (eds.) GW 2003. LNCS (LNAI), vol. 2915, pp. 409-420. Springer, Heidelberg (2004)

3. Karam, M., Schraefel, M.C.: A Taxonomy of Gestures in Human Computer Interactions. Technical Report, Electronics and Computer Science, University of Southampton (2005)

4. de la Barré, R., Chojecki, P., Leiner, U., Mühlbach, L., Ruschin, D.: Touchless InteractionNovel Chances and Challenges. In: Jacko, J.A. (ed.) HCI International 2009, Part II. LNCS, vol. 5611, pp. 161-169. Springer, Heidelberg (2009)

5. Burger, B., Ferrané, I., Lerasle, F., Infantes, G.: Two-handed Gesture Recognition and Fusion with Speech to Command a Robot. Auton. Robot. 32(2), 129-147 (2012)

6. Cerlinca, T.I., Pentiuc, S.G., Vatavu, R.D., Cerlinca, M.C.: Hand Posture Recognition for Human-Robot Interaction. In: 2007 Workshop on Multimodal Interfaces in Semantic Interaction, pp. 47-50. ACM, New York (2007)

7. Suay, H.B., Chernova, S.: Humanoid Robot Control Using Depth Camera. In: 6th International Conference on Human-Robot Interaction, pp. 401-402. ACM, New York (2011)

8. Rico, J., Brewster, S.: Usable Gestures for Mobile Interfaces: Evaluating Social Acceptability. In: SIGCHI Conference on Human Factors in Computing Systems, pp. 887-896. ACM, New York (2010)

9. Montero, C.S., Alexander, J., Marshall, M.T., Subramanian, S.: Would You Do That?: Understanding Social Acceptance of Gestural Interfaces. In: 12th International Conference on Human-Computer Interaction with Mobile Devices and Services, pp. 275-278. ACM, New York (2010)

10. Wobbrock, J.O., Morris, M.R., Wilson, A.D.: User-defined Gestures for Surface Computing. In: SIGCHI Conference on Human Factors in Computing Systems, pp. 1083-1092. ACM, New York (2009)

11. Gravill, J.I., Compeau, D.R., Marcolin, B.L.: Experience Effects on the Accuracy of Selfassessed User Competence. Inf. Manage. 43(3), 378-394 (2006)

12. Juutinen, S.: Emotional Obstacles of E-learning. Jyväskylä Studies in Computing, vol. 145. Jyväskylä University Printing House, Jyväskylä (2012)

13. Freeman, D., Hilliges, O., Sellen, A., O'Hara, K., Izadi, S., Wood, K.: The Role of Physical Controllers in Motion Video Gaming. In: Designing Interactive Systems Conference, pp. 701-710. ACM, New York (2012) 